\title{
Design of functionally graded composite structures in the presence of stress constraints
}

\author{
Robert Lipton \\ Department of Mathematics, Louisiana State University, Baton Rouge, LA 70803, USA \\ Received 4 April 2001
}

\begin{abstract}
In this work new homogenization results are used to introduce a methodology for the design of structural components made from composite materials in the presence of stress constraints. A numerical method is developed for finding functionally graded materials that provide the maximum torsional rigidity while keeping the mean square stress inside the composite structure below a prescribed level. @ 2002 Elsevier Science Ltd. All rights reserved.
\end{abstract}

Keywords: Stress constraints; Functionally graded material properties; Optimal design

\section{Introduction}

During the last few years several technologically important applications have benefited from the use of functionally graded composite materials (FGMs) (see Glasser, 1997; Koizumi, 1997). In many applications there is a separation of scales and the discrete entities forming up the microstructure exist on scales significantly smaller than the characteristic length scale of the loading. Under this hypothesis FGMs are modeled using effective thermophysical properties that depend upon features of the underlying microgeometry. The effective thermophysical properties are given by effective constitutive laws relating average flux to average gradient (see Markworth et al., 1995).

The primary problem of design of a FGM is the determination of the optimal spatial dependence for the composition. This type of problem has generated much interest in the engineering community and is the topic of a rapidly developing literature (see Markworth et al., 1995; Ootao et al., 2000). For many objective functions this type of problem has also received significant attention from both the applied mathematics and structural optimization communities in the 1980s and 1990s under the headings of the homogenization method for topology optimization and structural optimization (see Bendsoe and Kikuchi, 1988; Allaire and Kohn, 1993; Lurie and Cherkaev, 1986; Murat and Tartar, 1985; Cheng and Olhoff, 1981). Homogenization methods applied to the design of composites for optimal structural performance can be found in the

E-mail address: lipton@math.lsu.edu (R. Lipton). 
works of Sigmund and Torquato (1997), Fujii et al. (2001) and Diaz and Lipton (2000). This list is by no means complete and further references to the literature can be found in the books of Bendsoe (1995), Lurie (1993), Cherkaev and Kohn (1997) and Cherkaev (2000). In all of these works the problem of determining the optimal spatial dependence for the composition is obtained through the use of effective constitutive relations.

Motivated by the applications, this work treats the problem of maximizing structural stiffness subject to constraints on the stress. In many applications it is of central importance to control stress concentrations inside composite structural components. Regions of large stresses are most likely the first to exhibit failure during service. For this type of problem the effect of microscopic stresses needs to be accounted for. The concept of an effective constitutive law is by its self not sufficient to capture the effect of microstresses. This requires new modeling beyond the notion of effective thermophysical properties. The objective of this paper is to point out a new class of macroscopic tensors relevant to the modeling of microscopic stresses and to present a methodology for the numerical design of FGMs in the presence of stress constraints.

To fix ideas this article treats the problem of design of functionally graded reinforced shafts subject to torsion loading. However the approach presented here extends to fully three dimensional problems (see Lipton, 2001). The concept of a functionally graded material is introduced by considering a fiber reinforced shaft. Here the microstructure within the composite shaft consists of long reinforcement fibers with isotropic shear modulus $G_{\mathrm{f}}$ embedded in a more compliant matrix material with shear modulus $G_{\mathrm{m}}$. The shaft together with the fibers are right cylinders with generators along the $x_{3}$ axis and the cross-section of the reinforced shaft is a region $\Omega$ in the $x_{1}-x_{2}$ plane. The reinforcement fiber diameters are assumed to be of a size $\varepsilon$ that is much smaller than the diameter $d$ of the shaft cross-section $\Omega$. The graded material properties of the shaft are obtained by changing the local area fraction of fibers and other microgeometrical parameters across the cross-section of the shaft.

The composite shaft is subjected to torsion loading and the resulting stress tensor has the non-zero components $\tau_{13}$ and $\tau_{23}$. From the homogenization theory (Bensoussan et al., 1978; Murat and Tartar, 1997; Sanchez-Palencia, 1980), the actual in-plane stress $\tau=\left(\tau_{13}, \tau_{23}\right)$ is made up from macroscopic and microscopic components. We write $\tau=\tilde{\tau}+\tau_{\varepsilon}^{\mathrm{m}}$, where $\tilde{\tau}$ is the macrostress and $\tau_{\varepsilon}^{\mathrm{m}}$ is the microstress. Here the microstress depends upon the characteristic length of the microstructure given by $\varepsilon$. The macrostress $\tilde{\tau}$ can be written in terms of a homogenized stress potential $\tilde{\phi}$ that is a solution of

$$
-\operatorname{div}\left(S^{\mathrm{e}}(x) \nabla \tilde{\phi}\right)=1, \text { on } \Omega,
$$

and $\tilde{\phi}=0$ on the boundary of the cross-section. Here the homogenized compliance $S^{\mathrm{e}}(x)$ is associated with the local microstructure at $x$. The macrostress is given by $\tilde{\tau}=R \nabla \tilde{\phi}$, where $R$ is the $2 \times 2$ rotation matrix associated with a $90^{\circ}$ counterclockwise rotation. The coupling between the macroscopic stress $\tilde{\tau}$ and the local effects due to the microstructure is contained in the microstress $\tau_{\varepsilon}^{\mathrm{m}}$. The microstress $\tau_{\varepsilon}^{\mathrm{m}}$ is given by

$$
\tau_{\varepsilon}^{\mathrm{m}}=R\left(P^{\varepsilon}-I\right) R^{\mathrm{T}} \tilde{\tau}+\mathrm{O}(\varepsilon)
$$

where $P^{\varepsilon}$ is the "corrector matrix," (see Bensoussan et al., 1978; Murat and Tartar, 1997). It is evident that any meaningful stress constraint for FGMs needs to be applied to the actual stress in the composite not just the macroscopic part $\tilde{\tau}$. In this paper we consider a constraint on the mean square stress in the shaft crosssection given by

$$
\int_{\Omega}\left|\tilde{\tau}+\tau_{\varepsilon}^{\mathrm{m}}\right|^{2} \mathrm{~d} x_{1} \mathrm{~d} x_{2} \leqslant C
$$

where $C$ is the maximum tolerable value. The problem is to design a FGM for maximum torsional rigidity subject to the stress constraint given by (3). For small $\varepsilon$ we approximate the design problem by passing to 
the $\varepsilon=0$ limit. It is well known from homogenization theory (Murat and Tartar, 1985) that in the $\varepsilon=0$ limit, the torsional rigidity of the shaft is given by

$$
2 \int_{\Omega} \tilde{\phi} \mathrm{d} x_{1} \mathrm{~d} x_{2}
$$

On the other hand passing to the $\varepsilon=0$ limit in the stress constraint (3) requires new methods. This is due to the fact that that the coupling between the macroscopic stress and local effects due to microstructure do not decouple in this limit (see Lipton, 2001). It has recently been shown that

$$
\lim _{\varepsilon \rightarrow 0} \int_{\Omega}\left|\tilde{\tau}+\tau_{\varepsilon}^{\mathrm{m}}\right|^{2} \mathrm{~d} x_{1} \mathrm{~d} x_{2}=F(\tilde{\tau})
$$

where the "homogenized mean square stress" $F(\tilde{\tau})$ is given by

$$
F(\tilde{\tau})=\int_{\Omega}|\tilde{\tau}|^{2} \mathrm{~d} x_{1} \mathrm{~d} x_{2}+\int_{\Omega} R \sigma(x) R^{\mathrm{T}} \tilde{\tau} \cdot \tilde{\tau} \mathrm{d} x_{1} \mathrm{~d} x_{2}
$$

(see Lipton, 2001). Here $\sigma(x)$ is the covariance tensor introduced in Lipton (2000). The second term on the right hand side of (6) gives the contribution due to coupling between the macroscopic stress and local effects due to microstructure. The covariance tensor is a new type of effective or macroscopic property that depends only upon the microstructure. Setting $\alpha=1 /\left(2 G_{\mathrm{m}}\right)$ and $\beta=1 /\left(2 G_{\mathrm{f}}\right)$ the covariance is related to the effective compliance through the formula given by,

$$
\sigma(x)=\alpha^{-1}\left(S^{\mathrm{e}}(x)-\left(\alpha I+(\beta-\alpha) \partial_{\beta} S^{\mathrm{e}}(x)\right)\right) .
$$

Here $\partial_{\beta} S^{\mathrm{e}}(x)$ is the derivative of the effective compliance with respect to the material property $\beta$. Thus the covariance can be easily computed from the effective compliance if an explicit formula for the effective compliance is available. For the general case it is shown that $\sigma$ can be computed from the solutions of well defined microscopic equilibrium problems (see Lipton (2001); see also Lipton (2000) for the special case of periodic microstructures). We note here that $\sigma$ is a positive tensor so the coupling term in (6) is nonnegative. For the case of layered microstructures the methods developed earlier in Lipton and Velo (2002) and Velo (2000) allow one to correctly pass to the limit in (5).

To complete the discussion we suppose that we can fabricate a certain class of microstructures and we denote the set of associated effective compliances $S^{\mathrm{e}}$ by $A d$. It is evident from (4)-(6) that the optimal design of FGMs for maximum torsional rigidity with $d \gg \varepsilon$ is approximated by the problem

$$
\begin{aligned}
& \max _{S^{\mathrm{e}} \text { in } A d} 2 \int_{\Omega} \tilde{\phi} \mathrm{d} x_{1} \mathrm{~d} x_{2}, \\
& \text { subject to : } F(\tilde{\tau}) \leqslant C
\end{aligned}
$$

(see Lipton, 2001).

In the following sections we provide a numerical method for the optimal design of FGMs based on (8). Here the goal is to find the optimal macroscopic distribution for the microstructure. To fix ideas we consider microstructures given by configurations of fibers with identical circular cross-sections centered on a square lattice (see Fig. 1). The radii of the circular cross-sections are allowed to change on the macroscopic scale while all other geometric properties are held fixed. For this type of microstructure the effective compliance and covariance $S^{\mathrm{e}}$ and $\sigma$ are functions of the local area fraction $\theta(x)$ of the reinforcement fibers (see Section 3). Thus the design variable for this problem is the local area fraction of fibers. Here the local area fraction of the fiber phase is allowed to range between 0 and 0.5 . The graphs of $S^{\mathrm{e}}(\theta)$ and $\sigma(\theta)$ are plotted for the values $G_{\mathrm{m}}=1 \mathrm{GPa}$ and $G_{\mathrm{f}}=10 \mathrm{GPa}$ in Figs. 2 and 3 of Section 3. Writing $S^{\mathrm{e}}(x)=S^{\mathrm{e}}(\theta(x))$ 


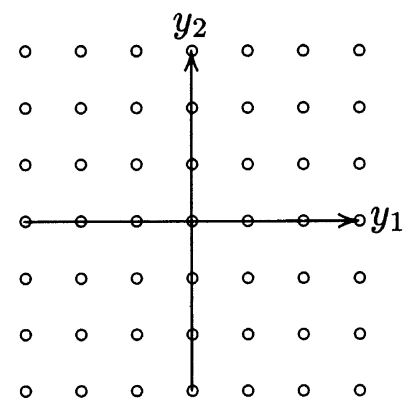

Fig. 1. A microstructure given by disks centered on a square lattice.

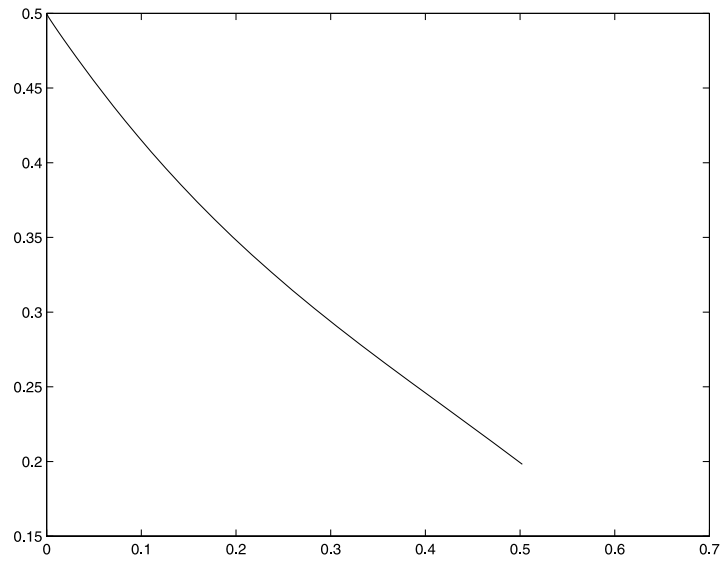

Fig. 2. Effective compliance $S^{\mathrm{e}}$ as a function of area fraction $\theta$.

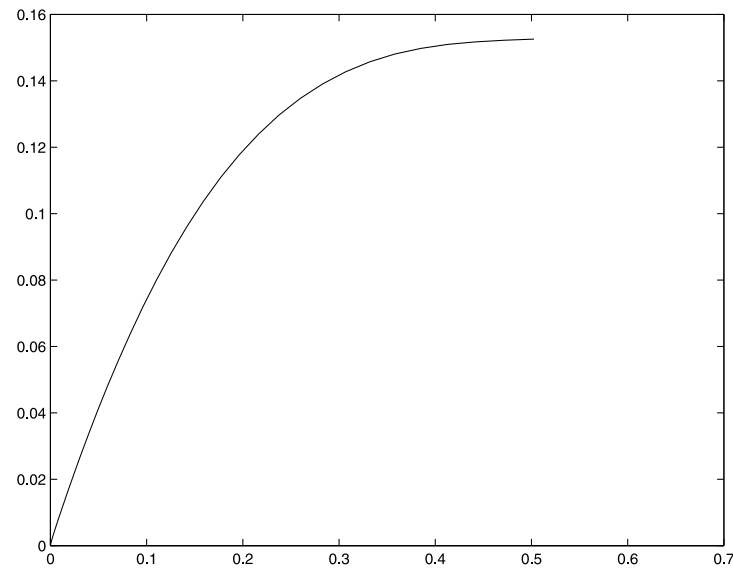

Fig. 3. Covariance $\sigma$ as a function of area fraction $\theta$. 
Table 1

Performance comparison

\begin{tabular}{|c|c|c|c|c|}
\hline & Area fraction $(\%)$ & Torsional rigidity & $F(\theta, \tilde{\tau})$ & Coupling term \\
\hline Stress constrained design & 23.7 & 1.78 & 6.32 & 0.63 \\
\hline Maximum rigidity design & 24.0 & 2.36 & 12.5 & 1.56 \\
\hline Minimum $F(\theta, \tilde{\tau})$ design & 23.2 & 1.35 & 3.7 & 0.2 \\
\hline
\end{tabular}

and $\sigma(x)=\sigma(\theta(x))$, the design problem becomes one of maximization over the area fraction $\theta(x)$ and is given by

$$
\begin{aligned}
& \max _{\theta} 2 \int_{\Omega} \tilde{\phi} \mathrm{d} x_{1} \mathrm{~d} x_{2}, \\
& \text { subject to: } F(\theta, \tilde{\tau})=\int_{\Omega}|\tilde{\tau}|^{2} \mathrm{~d} x_{1} \mathrm{~d} x_{2}+\int_{\Omega} R \sigma(\theta(x)) R^{\mathrm{T}} \tilde{\tau} \cdot \tilde{\tau} \mathrm{d} x_{1} \mathrm{~d} x_{2} \leqslant C, \\
& \text { where, } \tilde{\tau}=R \nabla \tilde{\phi} \text { and }-\operatorname{div}\left(S^{\mathrm{e}}(\theta(x)) \nabla \tilde{\phi}\right)=1, \text { on } \Omega .
\end{aligned}
$$

The optimal area fraction $\theta^{\text {opt }}(x)$ delivers the optimal spatial distribution of the fibers for the class of microstructures under consideration.

We solve the design problem given by (9) numerically. We compare our results with the optimal design when no constraint is placed on the stress and one seeks the FGM that maximizes the torsional rigidity subject to a resource constraint on the total area of the fiber phase. We conclude with a comparison to the design problem of minimizing the mean square stress subject to a resource constraint on the fibers. The performance specifications for each design are listed in Table 1. Our numerical solution of (9) shows that at the optimal design, about $10 \%$ of the stress constraint is in the coupling term

$$
\int_{\Omega} R \sigma\left(\theta^{\text {opt }}(x)\right) R^{\mathrm{T}} \tilde{\tau} \cdot \tilde{\tau} \mathrm{d} x_{1} \mathrm{~d} x_{2}
$$

(see Table 1).

To summarize, the treatment presented here is carried out in the asymptotic limit when the scale of the microstructure tends to zero relative to the length scale of the body forces and structural domain. The method presented here is suitable for design problems involving a very large number of small fibers. When the scale of the fiber microstructure is comparable to the design domain and the number of fibers is not too large in the graded direction the reader is referred to the work of Aboudi et al. (1997).

In Section 2 we give the mathematical arguments that illustrate the competition between design for maximum torsional rigidity and design for minimum stress.

\section{The competing requirements of maximum torsional rigidity and minimum stress}

In this section we demonstrate that optimizing over FGMs for maximum torsional rigidity competes with the requirement that

$$
F(\theta, \tilde{\tau})=\int_{\Omega}|\tilde{\tau}|^{2} \mathrm{~d} x_{1} \mathrm{~d} x_{2}+\int_{\Omega} R \sigma(\theta(x)) R^{\mathrm{T}} \tilde{\tau} \cdot \tilde{\tau} \mathrm{d} x_{1} \mathrm{~d} x_{2} \leqslant C,
$$

when $C$ is sufficiently small. We recall that the shear stiffness of the fibers is greater than that of the matrix, i.e., $G_{\mathrm{f}}>G_{\mathrm{m}}$. For the problem of fiber reinforced materials treated here it is easy to see that $S^{\mathrm{e}}(\theta)$ is a decreasing function of $\theta$. Indeed, for a unit period cell $Q$ consisting of a centered disk of radius $r_{1}$ 
surrounded by matrix material the associated effective property is denoted by $S^{\mathrm{e}}\left(\theta_{1}\right)$. For any constant vector $E$ the effective property $S^{\mathrm{e}}\left(\theta_{1}\right)$ is given by the variational principle (Bensoussan et al., 1978)

$$
S^{\mathrm{e}}\left(\theta_{1}\right) E \cdot E=\inf _{v \text { in } V} \int_{Q} S_{r_{1}}(x)|\nabla v+E|^{2} \mathrm{~d} x .
$$

Here the set $V$ is the set of all periodic square integrable functions with square integrable derivatives and $S_{r_{1}}(x)$ is the local compliance in the period cell taking the value $\beta$ in the matrix and $\alpha$ in the disk. Noting that $\beta<\alpha$ we see that the local compliance $S_{r_{2}}(x)$ associated with a disk of larger radius $r_{2}$ satisfies $S_{r_{2}}(x)<S_{r_{1}}(x)$ and $S^{\mathrm{e}}\left(\theta_{2}\right) E \cdot E<S^{\mathrm{e}}\left(\theta_{1}\right) E \cdot E$. This type of behavior is naturally found in the computations (see Fig. 2). For $\theta$ in the interval $[0,0.5]$ and for any vector $E$ it follows that

$$
S^{\mathrm{e}}(\theta) E \cdot E \geqslant S^{\mathrm{e}}(0.5) E \cdot E .
$$

We demonstrate that the torsional rigidity is greatest if $S^{\mathrm{e}}(\theta)=S^{\mathrm{e}}(0.5)$ everywhere in the shaft. We write

$$
\begin{aligned}
& T=\max _{\theta} 2 \int_{\Omega} \tilde{\phi} \mathrm{d} x_{1} \mathrm{~d} x_{2}, \\
& \text { subject to: }-\operatorname{div}\left(S^{\mathrm{e}}(\theta(x)) \nabla \tilde{\phi}\right)=1, \text { on } \Omega .
\end{aligned}
$$

We follow standard procedure and incorporate the differential equation

$$
-\operatorname{div}\left(S^{\mathrm{e}}(\theta(x)) \nabla \tilde{\phi}\right)=1
$$

into the objective functional to obtain

$$
T=2 \max _{\theta} \max _{\varphi}\left(2 \int_{\Omega} \varphi \mathrm{d} x_{1} \mathrm{~d} x_{2}-\int_{\Omega} S^{\mathrm{e}}(\theta) \nabla \varphi \cdot \nabla \varphi \mathrm{d} x_{1} \mathrm{~d} x_{2}\right),
$$

where $\varphi$ and its gradient are square integrable and $\varphi=0$ on the boundary of the shaft cross-section. Clearly for any $\varphi$ it is evident that

$$
-S^{\mathrm{e}}(\theta) \nabla \varphi \cdot \nabla \varphi \leqslant-S^{\mathrm{e}}(0.5) \nabla \varphi \cdot \nabla \varphi,
$$

and the most rigid shaft is obtained for $S^{\mathrm{e}}(\theta)=S^{\mathrm{e}}(0.5)$ everywhere in the shaft.

Next we will show that the quantity

$$
F(\theta, \tilde{\tau})=\int_{\Omega}|\tilde{\tau}|^{2} \mathrm{~d} x_{1} \mathrm{~d} x_{2}+\int_{\Omega} R \sigma(\theta(x)) R^{\mathrm{T}} \tilde{\tau} \cdot \tilde{\tau} \mathrm{d} x_{1} \mathrm{~d} x_{2}
$$

is smallest when there are no reinforcement fibers and the shaft is made from pure matrix material, i.e., when $S^{\mathrm{e}}(\theta)=S^{\mathrm{e}}(0)=\alpha$. Thus the requirement of keeping $F(\theta, \tilde{\tau})$ small is at odds with the fact that torsional rigidity is greatest when there is the highest allowable density of fibers everywhere in the shaft.

To see that $F(\theta, \tilde{\tau})$ is smallest when there are no fibers in the shaft we consider the stress potential $w$ associated with pure matrix material with compliance $\alpha$. For this case the covariance $\sigma$ vanishes. We also consider a stress potential $\tilde{\phi}$ associated with an FGM with compliance $S^{\mathrm{e}}(\theta)$ and associated covariance $\sigma(\theta)$. Standard arguments give the identities

$$
\begin{aligned}
& \int_{\Omega} \alpha \nabla w \cdot \nabla w \mathrm{~d} x_{1} \mathrm{~d} x_{2}=\int_{\Omega} w \mathrm{~d} x_{1} \mathrm{~d} x_{2}, \\
& \int_{\Omega} S^{\mathrm{e}}(\theta) \nabla \tilde{\phi} \cdot \nabla w \mathrm{~d} x_{1} \mathrm{~d} x_{2}=\int_{\Omega} w \mathrm{~d} x_{1} \mathrm{~d} x_{2} .
\end{aligned}
$$


We write

$$
\begin{aligned}
\int_{\Omega}|\nabla w|^{2} \mathrm{~d} x_{1} \mathrm{~d} x_{2} & =\frac{1}{\alpha} \int_{\Omega} \alpha \nabla w \cdot \nabla w \mathrm{~d} x_{1} \mathrm{~d} x_{2}=\frac{1}{\alpha} \int_{\Omega} w \mathrm{~d} x_{1} \mathrm{~d} x_{2} \\
& =\frac{1}{\alpha} \int_{\Omega} S^{\mathrm{e}}(\theta) \nabla \tilde{\phi} \cdot \nabla w \mathrm{~d} x_{1} \mathrm{~d} x_{2} \leqslant \frac{1}{\alpha} \sqrt{\int_{\Omega}\left|S^{\mathrm{e}}(\theta) \nabla \tilde{\phi}\right|^{2} \mathrm{~d} x_{1} \mathrm{~d} x_{2}} \sqrt{\int_{\Omega}|\nabla w|^{2} \mathrm{~d} x_{1} \mathrm{~d} x_{2}},
\end{aligned}
$$

where the last inequality follows from Cauchy's inequality. From this we see that

$$
\int_{\Omega}|\nabla w|^{2} \mathrm{~d} x_{1} \mathrm{~d} x_{2} \leqslant \frac{1}{\alpha^{2}} \int_{\Omega}\left|S^{\mathrm{e}}(\theta) \nabla \tilde{\phi}\right|^{2} \mathrm{~d} x_{1} \mathrm{~d} x_{2} .
$$

Elementary bounds for composite materials give that for any vector $\eta$

$$
\frac{1}{\alpha^{2}}\left|S^{\mathrm{e}}(\theta) \eta\right|^{2} \leqslant|\eta|^{2}
$$

and we find that

$$
\int_{\Omega}|\nabla w|^{2} \mathrm{~d} x_{1} \mathrm{~d} x_{2} \leqslant \int_{\Omega}|\nabla \tilde{\phi}|^{2} \mathrm{~d} x_{1} \mathrm{~d} x_{2} .
$$

Finally we recall that the stress is given by $\tau_{w}=R \nabla w$ where $R R^{\mathrm{T}}=I$ and since $\sigma(0)=0$ we see that for no fibers the quantity (16) is just

$$
\int_{\Omega}|\nabla w|^{2} \mathrm{~d} x_{1} \mathrm{~d} x_{2}=\int_{\Omega}\left|\tau_{w}\right|^{2} \mathrm{~d} x_{1} \mathrm{~d} x_{2}
$$

Similarly $\tilde{\tau}=R \nabla \tilde{\phi}$ and since $\sigma(\theta) \geqslant 0$ we recover the required inequality

$$
F\left(0, \tau_{w}\right)=\int_{\Omega}\left|\tau_{w}\right|^{2} \mathrm{~d} x_{1} \mathrm{~d} x_{2} \leqslant F(\theta, \tilde{\tau}) .
$$

\section{The effective compliance and covariance tensors}

In this section we present the microscopic equilibrium equation used in the computation of effective compliance and covariance tensors for a periodic array of fibers. We suppose that the fiber cross-sections are disks centered on a square lattice (see Fig. 1). We compute the effective compliance and covariance for area fractions of disks ranging from 0 to 0.5 . The shear moduli of the fibers and matrix are 10 and $1 \mathrm{GPa}$ respectively. The effective properties are given in terms of the solution of the following "microscopic" problem in the unit period cell $Q$ in the $y_{1}-y_{2}$ plane. We look for solutions of the form $u^{j}=v^{j}+y_{j}, j=1,2$, where $v^{j}$ is $Q$ periodic and

$$
\begin{aligned}
& \alpha \Delta u^{j}=0 \text { in the fiber, } \\
& \beta \Delta u^{j}=0 \text { in the matrix. }
\end{aligned}
$$

Here $\alpha=1 /\left(2 G_{\mathrm{f}}\right), \beta=1 /\left(2 G_{\mathrm{m}}\right)$ where $G_{\mathrm{f}}=10 \mathrm{GPa}$ and $G_{\mathrm{m}}=1 \mathrm{GPa}$. The solution is continuous across the interface and

$$
\alpha \partial_{n} u_{\left.\right|_{\mathrm{f}} ^{j}}^{j}=\beta \partial_{n} u_{\left.\right|_{\mathrm{m}}}^{j},
$$

where $\partial_{n}$ is the normal derivative at the interface with normal vector pointing into the matrix phase and the subscripts $\mathrm{m}$ and $\mathrm{f}$ indicate the side of the interface where the normal derivative is evaluated. We denote the 
unit vector in the $j$ th direction by $e^{j}, j=1,2$ and it is well known (Bensoussan et al., 1978; Sanchez-Palencia, 1980) that the effective compliance tensor is given by

$$
S_{i j}^{\mathrm{e}}=\int_{Q} S(x) \nabla u^{i} \cdot e^{j} \mathrm{~d} x_{1} \mathrm{~d} x_{2} .
$$

Here $S(x)=\alpha$ in the fiber and $\beta$ in the matrix. The covariance is given by

$$
\sigma_{i j}=\int_{Q} \nabla v^{i} \cdot \nabla v^{j} \mathrm{~d} x_{1} \mathrm{~d} x_{2}
$$

(see Lipton, 2000, 2001). The tensors $S^{\mathrm{e}}$ and $\sigma$ are functions of the fiber radii $r$ or equivalently functions of the area fraction $\theta=\pi r^{2}$. For the square period cell it is easily seen that $S^{\mathrm{e}}$ and $\sigma$ are invariant under the group of rotations leaving the square invariant. Thus they are isotropic tensors and we write $S^{\mathrm{e}}=S^{\mathrm{e}}(\theta) I$ and $\sigma=\sigma(\theta) I$, where $I$ is the $2 \times 2$ identity. The functions $S^{\mathrm{e}}(\theta)$ and $\sigma(\theta)$ are computed numerically for thirty values over the interval $0 \leqslant \theta<0.5$ and are plotted in Figs. 2 and 3. These data points are interpolated using 5 th order polynomials.

\section{Solution algorithm}

The optimal FGM for (9) is found using a steepest decent method. The design domain is a square of side length two. The design variable is the local area fraction of fibers $\theta=\theta(x)$. For the numerical experiments the area fraction is discretized and represented as a piecewise constant function. The design domain is divided into 400 square subdomains or cells each of side length $\kappa=0.1$. The local area fraction is allowed to take different values in each cell. All computations are done in terms of the stress potential $\tilde{\phi}$. To this end we note that under the relation $\tilde{\tau}=R \nabla \tilde{\phi}$ the stress constraint becomes

$$
\int_{\Omega}(1+\sigma(\theta))|\nabla \tilde{\phi}|^{2} \mathrm{~d} x_{1} \mathrm{~d} x_{2} \leqslant C
$$

We include the stress constraint through the addition of a penalty term and write the augmented objective function $L(\theta, \tilde{\phi})$ as

$$
L(\theta, \tilde{\phi})=-2 \int_{\Omega} \tilde{\phi} \mathrm{d} x_{1} \mathrm{~d} x_{2}+\ell \int_{\Omega}(1+\sigma(\theta))|\nabla \tilde{\phi}|^{2} \mathrm{~d} x_{1} \mathrm{~d} x_{2} .
$$

The design problem is given by

$$
\begin{aligned}
& \min _{\theta} L(\theta, \tilde{\phi}) \\
& \text { where, }-\operatorname{div}\left(S^{\mathrm{e}}(\theta) \nabla \tilde{\phi}\right)=1 \text {, on } \Omega, \quad \tilde{\phi}=0, \text { on } \partial \Omega .
\end{aligned}
$$

To compute sensitivities we follow standard procedure and introduce the adjoint field $\lambda$. Here $\lambda$ is the solution of

$$
-\operatorname{div}\left(S^{\mathrm{e}}(\theta) \nabla \lambda\right)=1+2 \ell \operatorname{div}((1+\sigma(\theta)) \nabla \tilde{\phi}),
$$

where $\lambda=0$ on the boundary of the shaft. For $\delta \ll 1$, the change in the stress potential $\tilde{\phi}$ due to a small perturbation $\delta \hat{\theta}$ in the local density $\theta$ is written as $\hat{\phi}$ and

$$
-\operatorname{div}\left(S^{\mathrm{e}}(\theta) \nabla \hat{\phi}\right)=\operatorname{div}\left(\hat{\theta} \partial_{\theta} S^{\mathrm{e}}(\theta) \nabla \tilde{\phi}\right),
$$


where $\hat{\phi}=0$ on $\partial \Omega$. Taking the first variation of $L(\theta, \tilde{\phi})$ with respect to the design variable $\theta$ gives to lowest order

$$
\Delta L=-\delta \int_{\Omega} \hat{\phi} \mathrm{d} x_{1} \mathrm{~d} x_{2}+\delta \ell\left(\int_{\Omega} 2(\nabla \tilde{\phi}+\sigma(\theta) \nabla \tilde{\phi}) \cdot \nabla \hat{\phi} \mathrm{d} x_{1} \mathrm{~d} x_{2}+\int_{\Omega} \hat{\theta} \partial_{\theta} \sigma(\theta)|\nabla \tilde{\phi}|^{2} \mathrm{~d} x_{1} \mathrm{~d} x_{2}\right) .
$$

Application of (1), (32) and (33) gives

$$
\Delta L=\delta \int_{\Omega} \hat{\theta}\left(\partial_{\theta} S^{\mathrm{e}}(\theta) \nabla \tilde{\phi} \cdot \nabla \lambda+\ell \partial_{\theta} \sigma(\theta) \nabla \tilde{\phi} \cdot \nabla \tilde{\phi}\right) \mathrm{d} x_{1} \mathrm{~d} x_{2}
$$

Denoting the $i$ th cell by $\Omega_{i}$ the restriction of the piecewise constant perturbation $\hat{\theta}$ on that set is denoted by $\hat{\theta}_{i}$. The choice of $\hat{\theta}$ that renders $\delta L$ the most negative is given by

$$
\hat{\theta}_{i}=-\frac{1}{\left|\Omega_{i}\right|} \int_{\Omega_{i}}\left(\partial_{\theta} S^{\mathrm{e}}(\theta) \nabla \tilde{\phi} \cdot \nabla \lambda+\ell \partial_{\theta} \sigma(\theta) \nabla \tilde{\phi} \cdot \nabla \tilde{\phi}\right) \mathrm{d} x_{1} \mathrm{~d} x_{2}, \quad \text { on each } \Omega_{i}
$$

For this choice the objective is non-increasing, i.e.,

$$
\Delta L=-\delta \sum_{i=1}^{400} \hat{\theta}_{i}^{2}\left|\Omega_{i}\right|
$$

We outline the steepest decent method for this problem. For a given $\theta$ we solve for $\tilde{\phi}$ and $\lambda$ using the system of equations given by (1) and (32). The design variable $\theta$ is updated according to $\theta_{\text {new }}=\theta_{\text {old }}+\delta \hat{\theta}$ where $\hat{\theta}$ is chosen according to (36). This algorithm is guaranteed to converge due the monotonicity of the objective under our choice of perturbation. In the computations the stress potential and adjoint field are solved using standard triangular finite elements. The mesh for the stress potential and adjoint field is finer than the size of the cells. Roughly 25 elements per cell are used in the computations.

\section{Computational design for optimal FGMs}

In this section we present numerical results for the stress constrained problem given by (9). We compare these results with the optimal FGM that maximizes the torsional rigidity in the absence of stress constraints. For completeness we also compare with the design problem of minimizing the mean square stress subject to a resource constraint on the fibers. Here the design domain is a square cross-section of side length two. The design variable is the local area fraction $\theta(x)$. It is discretized over 400 square subdomains of side length 0.1 and is given by a piecewise constant function taking values in the interval $0 \leqslant \theta \leqslant 0.5$.

For the stress constrained problem (9) we set the stress constraint to be $C=6.33$. The total area fraction of the design domain occupied by the fibers for this numerical experiment is $23.7 \%$. The goal is to find the optimal distribution of the local area fraction of the fibers denoted by $\theta^{\text {opt }}$. At the optimal design the numerical results show that the homogenized mean square stress $F\left(\theta^{\text {opt }}, \tilde{\tau}\right)$ is 6.32 . Roughly $10 \%$ of this is in the coupling term, i.e.,

$$
\int_{\Omega} R \sigma\left(\theta^{\mathrm{opt}}(x)\right) R^{\mathrm{T}} \tilde{\tau} \cdot \tilde{\tau} \mathrm{d} x_{1} \mathrm{~d} x_{2}=0.63
$$

The macroscopic term in the stress constraint is

$$
\int_{\Omega}|\tilde{\tau}|^{2} \mathrm{~d} x_{1} \mathrm{~d} x_{2}=5.69
$$

The torsional rigidity for this design is 1.78 . We give a gray scale plot of $\theta^{\text {opt }}$ over the design domain in Fig. 4. Here the darkest regions are where $\theta=0.5$ and the lightest regions correspond to $\theta=0$. The design 


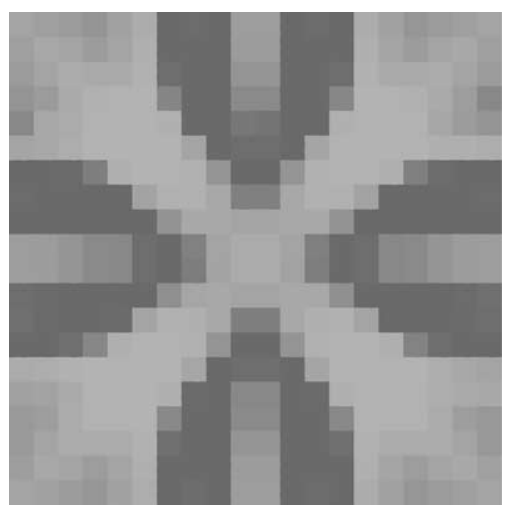

Fig. 4. Stress constrained design for maximum torsional rigidity.

consists of four spokes radiating out from the center of the shaft. In Fig. 5 a graph of $\theta^{\text {opt }}$ is plotted through the cross-section along the line $x_{1}=0,-1<x_{2}<1$. Next we compute the distribution of area fraction $\theta^{\mathrm{opt}}$ that maximizes the torsional rigidity in the absence of stress constraints. We fix the total area fraction of the design domain occupied by the fibers to be $24.0 \%$. This is nearly the same total area fraction of fibers used in the stress constrained design. A gray scale plot of $\theta^{\text {opt }}$ for this design problem is given in Fig. 6. For this problem the numerics give a design with a torsional rigidity of 2.36 and the homogenized mean square stress $F\left(\theta^{\text {opt }}, \tilde{\tau}\right)$ is 12.5 . From Fig. 6 it is clear that this design emulates an annular cross-section. We point out that the mean square stress for this design is nearly twice that of the stress constrained design while the rigidity of this design offers a 30\% improvement over the rigidity associated with the stress constrained design.

Last we examine the design that minimizes the homogenized mean square stress $F(\theta, \tilde{\tau})$. Here we fix the total area fraction of the design domain occupied by the fibers to be $23.2 \%$. The homogenized mean square stress for this design problem is 3.7. A gray scale plot of $\theta^{\text {opt }}$ is given in Fig. 7. The results of the three design problems are listed in Table 1.

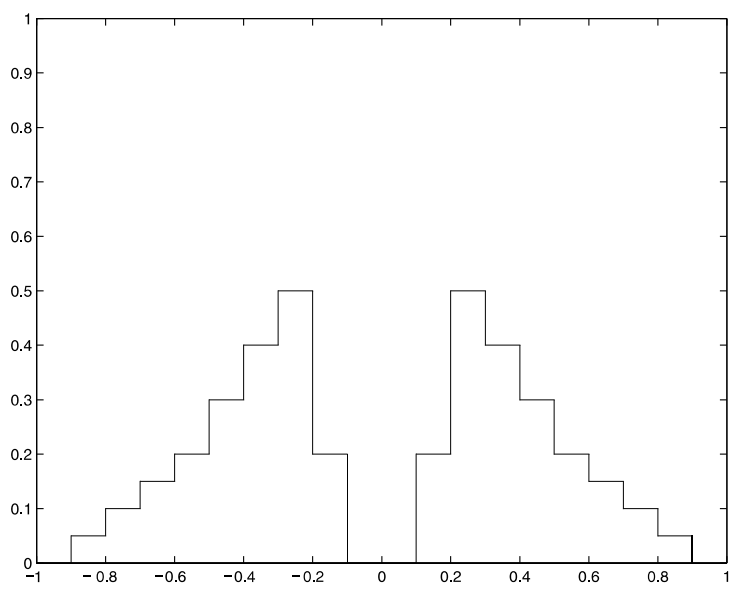

Fig. 5. Plot of $\theta^{\text {opt }}$ across the cross-section. 


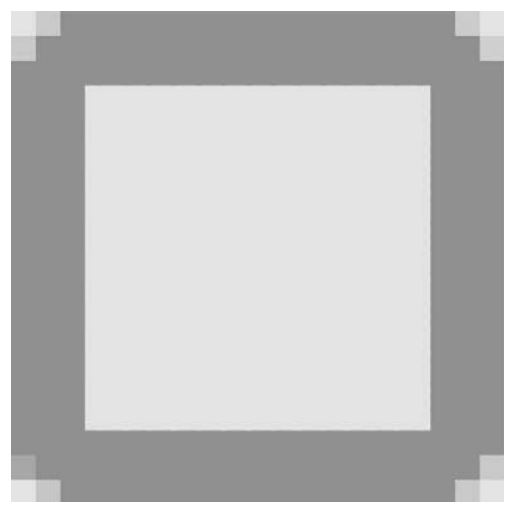

Fig. 6. Design for maximum rigidity without stress constraints.

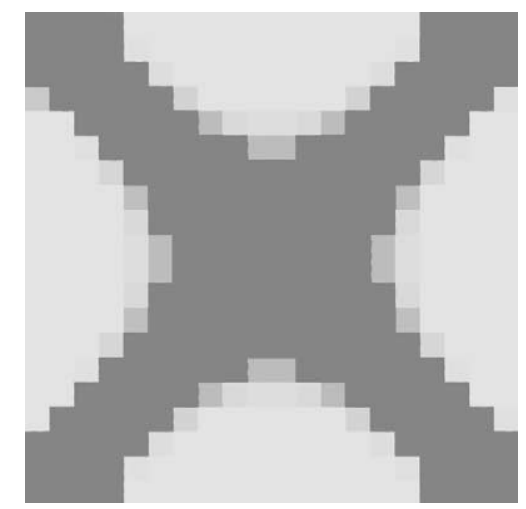

Fig. 7. Design for minimum homogenized mean square stress.

\section{Acknowledgements}

The author would like to thank Ani Velo for her comments. This research effort is sponsored by NSF through grant DMS-0072469 and by the Air Force Office of Scientific Research, Air Force Materiel Command USAF, under grant number F49620-99-1-0009. The US Government is authorized to reproduce and distribute reprints for governmental purposes notwithstanding any copyright notation thereon. The views and conclusions herein are those of the authors and should not be interpreted as necessarily representing the official policies or endorsements, either expressed or implied of the Air Force Office of Scientific Research or the US Government.

\section{References}

Aboudi, J., Pindera, M.J., Arnold, S.M., 1997. Microstructural optimization of functionally graded composites subjected to thermal gradient via the coupled higher-order theory. Composites Part B 28B, 93-108.

Allaire, G., Kohn, R.V., 1993. Optimal design for minimum weight and compliance in plane stress using extremal microstructure. Eur. J. Mech. 12, 839-878.

Bendsoe, M.P., 1995. Optimization of Structural Topology, Shape, and Material. Springer-Verlag, Berlin. 
Bendsoe, M.P., Kikuchi, N., 1988. Generating optimal topologies in structural design using a homogenization method. Comput. Methods Appl. Mech. Eng. 71, 197-224.

Bensoussan, A., Lions, J.L., Papanicolaou, G., 1978. Asymptotic analysis for periodic structures. In: Studies in Mathematics and its Applications, vol. 5. North-Holland, Amsterdam.

Cheng, K.T., Olhoff, N., 1981. An investigation concerning optimal design of solid elastic plates. Int. J. Solids Struct. 17, $305-323$.

Cherkaev, A., 2000. Variational Methods for Structural Optimization. Springer Verlag, New York, NY.

Cherkaev, A., Kohn, R.V., 1997. Topics in the mathematical modelling of composite materials. In: Cherkaev, A., Kohn, R. (Eds.), Progress in Nonlinear Differential Equations and their Applications, vol. 31. Birkhäuser Boston, Inc., Boston, MA.

Diaz, A.R., Lipton, R., 2000. Optimal material layout for three-dimensional elastic structures subject to multiple loads. Mech. Struct. Mach. 28, 219-236.

Fujii, D., Chen, B.C., Kikuchi, N., 2001. Composite material design of two-dimensional structures using the homogenization design method. Int. J. Numer. Methods Eng. 50, 2031-2051.

Glasser, A.M., 1997. The use of transient FGM interlayers for joining advanced ceramics. Composites Part B $28 \mathrm{~B}, 71-84$.

Koizumi, M., 1997. FGM activities in Japan. Composites Part B 28B, 1-4.

Lipton, R., 2000. Optimal bounds on electric field fluctuations for random composites. J. Appl. Phys. 80, 4287-4293.

Lipton, R., 2001. Relaxation through homogenization for optimal design problems with gradient constraints. J. Optim. Theory Applicat., in press.

Lipton, R., Velo, A., 2002. Optimal design of gradient fields with applications to electrostatics. Nonlinear Partial Different. Equat. Applicat.: Seminar du College de France, J.L. Lions Series in Applied Mathematics. P.G. Ciarlet et P.L. Lions, (Eds.), ElsevierGauthier-Villars, to appear.

Lurie, K., 1993. Applied Optimal Control Theory of Distributed Systems. Plenum Press, New York and London.

Lurie, K.A., Cherkaev, A.V., 1986. Effective characteristics of composite materials and the optimal design of structural elements. Uspekhi Mekhaniki (Adv. Mech.) 9 (2), 3-81.

Markworth, A.J., Ramesh, K.S., Parks, W.P., 1995. Modelling studies applied to functionally graded materials. J. Mater. Sci. 30, 2183-2193.

Murat, F., Tartar, L., 1985. Calcul des variations et homogénéisation. Les Méthodes de l'Homogénéisation: Théorie et Applications en Physique. Ecole d'Eté d'Analyse Numérique CEA-EDF-INRA (Bréau-sans-Nappe, 1983), Collection de la Direction des Études et Recherches d'Electricité de France, vol. 57, Eyrolles, Paris, pp. 319-369.

Murat, F., Tartar, L., 1997. H-convergence. In: Cherkaev, A., Kohn, R.V. (Eds.), Topics in the Mathematical Modeling of Composite Materials. Berkhauser, Boston, pp. 21-43.

Ootao, Y., Tanigawa, Y., Ishimaru, O., 2000. Optimization of material composition of functionally graded plate for thermal stress relaxation using a genetic algorithim. J. Therm. Stresses 23, 257-271.

Sanchez-Palencia, E., 1980. Nonhomogeneous media and vibration theory. In: Lecture Notes in Physics, vol. 127. Springer Verlag, Berlin.

Sigmund, O., Torquato, S., 1997. Design of materials with extreme thermal expansion using a three-phase topology optimization method. J. Mech. Phys. Solids 45, 1037-1067.

Velo, A., 2000. Optimal Design of Gradient Fields with Applications to Electrostatics. Ph.D. Thesis, Department of Mathematical Sciences, Worcester Polytechnic Institute. 